\title{
Salivary Amino Acids Determination and Their Changes in Vision Stress Experiments
}

\author{
Wei Tang ${ }^{1}$, Xuesong $\mathrm{Li}^{1}$, Xiaoxiao $\mathrm{Wu}^{1}$, Yu Wang ${ }^{1}$, Xuejun Kang ${ }^{1,2^{*}}$ \\ ${ }^{1}$ Key Laboratory of Child Development and Learning Science of Ministry of Education, \\ Research Center for Learning Science, Southeast University, Nanjing, China \\ ${ }^{2}$ Suzhou Key Laboratory of Enviroment and Biosafety, Southeast University, Suzhou, China \\ Email: "xjkang64@163.com
}

Received October 2013

\begin{abstract}
Amino acid neurotransmitters represent a major class of compounds that are involved in neuronal communication at CNS synapses, which can provide the basis for a variety of disease diagnosis and treatments and the study of the mechanism of mental illness. An analytical method for the determination of several amino acids in saliva was established with reversed-phase high performance liquid chromatography (RPHPLC) with UV detector. About ten kinds of amino acids were detected in saliva. Nine subjects have participated in the stress experiments which have undergone a 50-min three-dimensional cartoon watching. The result of the experiment has proved that four kinds of salivary amino acids respond to the vision stress experiment obviously.
\end{abstract}

Keywords: Amino Acids; RP-HPLC; Stress Experiments

\section{Introduction}

Stress research in human health and diseases has occupied a prominent position. According to the literature survey, about 75 to $90 \%$ of the diseases have a connection with the activation of stress mechanisms. Many factors cause stress, such as mechanical trauma, undercooling, overheating, food poisoning, psychological fear, depression, heavily mental tension and so on. Stress has a variety of assessment methods. Many indicators of stress response can really and effectively show human being's physiological stress response system, such as cortisol, catecholamines and so on. Among them the amino acid neurotransmitters are important stress markers. Glutamate, aspartate, $\gamma$-aminobutyric acid, glycine and taurine and many kinds of amino acids are important neurotransmitters in brain. Glutamate and aspartate are considered to be excitatory amino acids; while $\gamma$ aminobutyric acid, glycine and taurine are considered to be inhibitory amino acids. They are important materials which can regulate the body's physiological activities. Thus separating and determining the content of biological tissue's amino acid neurotransmitters can provide a basis for the diagnosis and the treatment of many diseases and mental illness' mechanisms. As a result, the determination and study of stress amino acids are really important (Xuejun Kang, Jing Xiao, Xiao Huang, \& Zhongze Gu, 2006).

Amino acids are regarded as important nutrients which can directly involve brain protein synthesis and metabolism, (Spencer GS, 1994) play an important regulatory role on the brain function and psychological behavior. The researches of amino acids and nervous system's function can be traced back to the forties and fifties last century. In recent years, neurotransmitters and their receptors, neurophysiological and biochemical pharmacology research also have many new developments (Yan SL, Zhao G, \& Liu YL, 2003). Studies have shown that some ami-

*Corresponding author. no acids are involved in learning and memorizing and some other complex nervous activities (Jones DP, Carlson JL, \& Samiec PS, 1998).

Kodama and co-workers found that concentrations of glycine, alanine, threonine and histidine in the saliva are slightly elevated, whereas those of glutamic acid and lysine are reduced during the examination. The enhanced ratio of glycine/glutamic acid may reflect the change from the excited state to the depressed state (Nakamura Y, Kodama H, Satoh T, Adachi K, Watanabe S, Yokote Y et al., 2010). Our group's researches imply that L-histidine in saliva may be another index in correlation with human stress (Jing Sun, Xuejun Kang, Yuqin Ma, Liqin Chen, Zijian Qu, Zhongze Gu, \& Zuhong Lu, 2009) (Jing Sun, Shenglan Zheng, Yu Wang, Xuejun Kang, Zhongze Gu, \& Zuhong Lu, 2010).

The aim of the present study was to use HPLC to determine amino acids, and to evaluate the response of some salivary amino acids on the learning process of people submitted computer vision stress.

\section{Experimental}

\section{Chemicals and Materials}

Methanol (chromatographic purity) was from Chengdu Xin$\mathrm{du}$ area of the industrial development zone. (Chengdu, China). Acetonitrile was from Shanghai Ludu Chemical Reagent Co., Ltd. (Shanghai, China). Triethyl ammonium was from Sinopharm Chemical Reagent Co., Ltd. Tetrabutyl ammonium hydroxide was from Shanghai Kefeng Industry Co., Ltd. (Shanghai, China). Dansyl chloride and amino acid standards were from Sigma (St. Louis, MO). Acetone was from Shanghai Lingfeng Chemical Reagent Co., Ltd. (Shanghai, China). Potassium bicarbonate, potassium hydroxide and glacial acetic acid were from Nanjing Chemical Reagent Co., Ltd. (Jiangsu Province, China). Triply distilled water was used for all studies. 


\section{Instrument and Chromatographic Conditions}

The instruments used are as follows: LC-20AD HPLC; SPD10AD UV detector (Shimadzu Corporation); Shimadzu chromatography workstation; Shimadzu C18 column $(4.6 \times 150 \mathrm{~mm}$, $5 \mu \mathrm{m}$ ); GL-20B high speed refrigerated centrifuge (Shanghai Anting Scientific Instrument Factory); GL-88B vortex mixer (Jiangsu Haimen Kirin Medical Instrument factory); PHS-2C $\mathrm{pH}$ meter; METTLERAT electronic analytical balance; BF2500 positive and negative pressure oil-free vacuum pump (Shanghai Rate Nako Trading Co., Ltd.); electric constant temperature water bath.

The mobile phase was 35:1.5:80 methanol/acetonitrile/water (V/V/V) containing $1.3 \%$ triethylammonia and $1.1 \%$ tetrabutyl ammonium hydroxide. The mobile phase was adjusted to $\mathrm{PH}$ 2.53 with concentrated sulfuric acid, and then was filtrated with a $0.45 \mu \mathrm{m}$ microporous organic membrane. The UV detection wavelength was $221 \mathrm{~nm}$. The flow rate of the mobile phase was $1.5 \mathrm{ml} / \mathrm{min}$. The injection volume was $20 \mu \mathrm{l}$. The column temperature was $35^{\circ} \mathrm{C}$.

\section{Experimental Procedure}

Accurately weighed thirteen kinds of amino acids, glycine, glutamate, glutamine, Aspartate, taurine, arginine, hydroxyproline, threonine, ornithine, lysine, serine, alanine, $\gamma$-aminobutyric acid and mixed with triply distilled water to prepare $1.0 \mathrm{mg} / \mathrm{ml}$ standard solution. These solutions were diluted daily to working concentrations with triply distilled water. The dansyl chloride solution was prepared just before derivatization by dissolving $200 \mathrm{mg}$ of Dns in $10 \mathrm{ml}$ of acetone.

Subjects were 9 graduates undergoing a 50-min three-dimensional cartoon watching. Saliva samples were spit in a centrifuge tube after rinsing the mouth with water. Participants in this experiment provided three salivary samples, 20-min before the vision stress experiment, at the time of the process end and 20-min post-process.

$50 \mu$ of the standard solution or the test saliva sample was added to $50 \mu \mathrm{l}$ of buffer solution of $2 \mathrm{~mol} / \mathrm{l} \mathrm{KOH}-\mathrm{KHCO}_{3}(\mathrm{PH}$ $=9.8$ ) and $20 \mathrm{mg} / \mathrm{ml}$ of dansyl chloride (acetone) solution $(50 \mu \mathrm{l})$. After capping the tube and mixing vigorously for $5 \mathrm{~s}$, the mixture was reacted in the dark at $60^{\circ} \mathrm{C}$ in a water bath for $60 \mathrm{~min}$. Then, an aliquot $(20 \mu \mathrm{l})$ of acetic acid was added into the tube to stop the reaction. The mixture was centrifuged at $10,000 \times \mathrm{g}$ for $5 \mathrm{~min}$. The $20 \mu \mathrm{l}$ supernatant of the reaction mixture was injected into the HPLC system.

\section{Results and Discussion}

\section{Method Validation}

Good linearity was observed over the concentration range of $0.5 \sim 100 \mu \mathrm{g} / \mathrm{ml}$, and the correlation coefficient of 13 kinds of amino acids is from 0.9934 to 0.9999 . The LOD is $0.1-0.5$ $\mu \mathrm{g} / \mathrm{ml}$. The precision of the method was evaluated as RSD $0.3 \%$ - 8.8\% ( $n=6)$. The content of each amino acid in saliva is in the range of $0.3-5.0 \mu \mathrm{g} / \mathrm{ml}$. The chromatograms of blank, the amino acid standard mixture, and the samples are shown as A, $\mathrm{B}, \mathrm{C}$ in Figures 1 and 2.

\section{The Response of Salivary Amino Acids to Vision Stress}

Saliva was collected before and after the stress experiment,
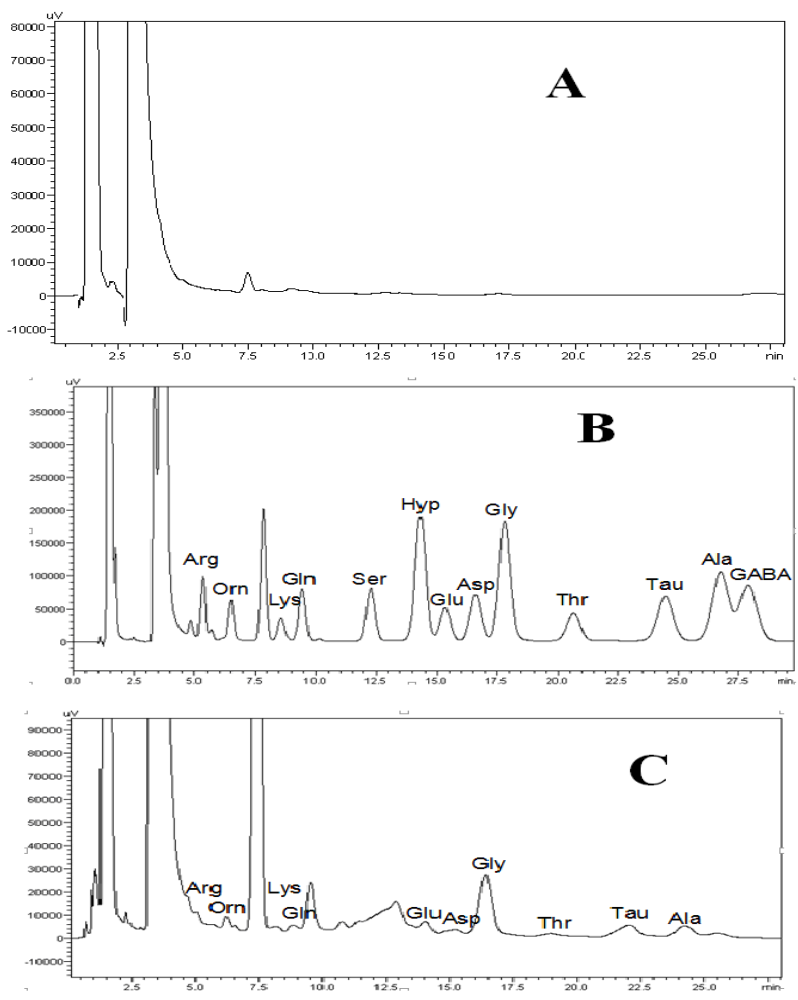

Figure 1.

Typical HPLC separations of target amino acids; (A) A blank (derivatization solution with no analyte); (B) 13 amino acid standards; (C) Human saliva sample.

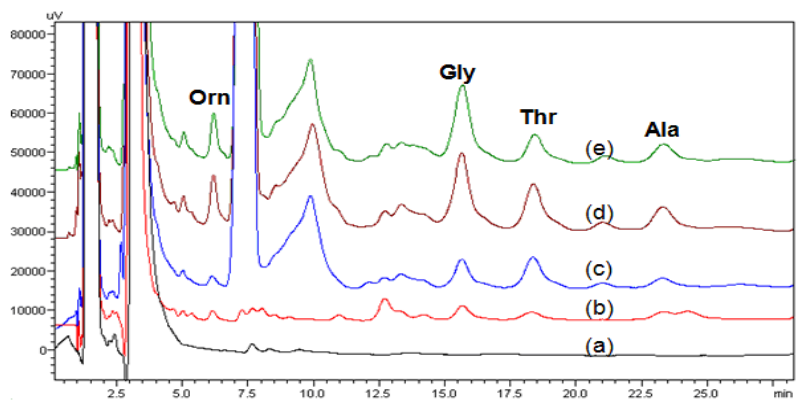

Figure 2.

Chromatograms of salivary amino acids of one subject. (a) Blank; (b) 5 $\mu \mathrm{g} / \mathrm{ml}$ amino acid standard solution; (c) Sample at 20-min before the vision stress experiment; (d) Sample at the end of vision stress experiment; (e) Sample at 20-min after the vision stress experiment.

respectively, and amino acid concentrations were measured by using HPLC. The effect of stress on the amino acid concentration was significantly observed only for glycine, threonine, alanine, ornithine. At the end of the vision stress experiment, those four amino acids were increased, whereas they were reduced at 20-min after the stress. The results were shown in Table 1 and Figure 3.

\section{Conclusion}

The present study demonstrated that thirteen kinds of amino acids can be detected in the human saliva by the method we have developed. Salivary glycine, threonine, alanine, ornithine 
Table 1.

The content of four kinds of amino acids in saliva of subjects $(\mu \mathrm{g} / \mathrm{ml})$.

\begin{tabular}{cccccccccccc}
\hline \multicolumn{2}{c}{ Subject } & 1 & 2 & 3 & 4 & 5 & 6 & 7 & 8 & 9 \\
\hline \multirow{4}{*}{ Gly } & Before & 2.84 & 0.79 & 3.35 & 7.36 & 11.04 & 7.32 & 3.72 & 3.00 & 4.54 \\
& End & 2.95 & 3.78 & 10.36 & 7.78 & 22.20 & 8.42 & 10.47 & 9.19 & 8.96 \\
& 20'post & 2.91 & 1.42 & 6.58 & 4.11 & 13.43 & 8.32 & 9.36 & 8.49 & 3.11 \\
& Before & 1.68 & 1.44 & 5.50 & 8.49 & 4.05 & 3.74 & 1.77 & 5.66 & 6.97 \\
\multirow{3}{*}{ Thr } & End & 1.85 & 4.48 & 5.74 & 19.53 & 9.66 & 5.04 & 3.94 & 8.20 & 7.43 \\
& 20’post & 1.48 & 3.00 & 2.91 & 9.09 & 3.33 & 2.87 & 3.41 & 4.52 & 6.86 \\
& Before & 1.16 & 0.46 & 1.86 & 2.27 & 4.45 & 3.19 & 1.63 & 1.79 & 2.83 \\
\multirow{4}{*}{ Ala } & End & 0.95 & 1.78 & 3.15 & 5.57 & 6.36 & 5.12 & 1.77 & 4.47 & 2.86 \\
& 20’post & 1.12 & 0.88 & 1.64 & 2.60 & 5.62 & 3.91 & 1.49 & 3.26 & 2.77 \\
& Before & 1.95 & 0.97 & 1.66 & 5.66 & 17.50 & 5.29 & 6.07 & 5.76 & 19.40 \\
\multirow{2}{*}{ Orn } & End & 2.90 & 5.33 & 6.69 & 18.54 & 26.37 & 5.65 & 10.88 & 28.24 & 25.50 \\
& 20'post & 2.38 & 2.05 & 4.64 & 5.46 & 8.69 & 3.80 & 1.60 & 25.60 & 21.20 \\
\hline
\end{tabular}
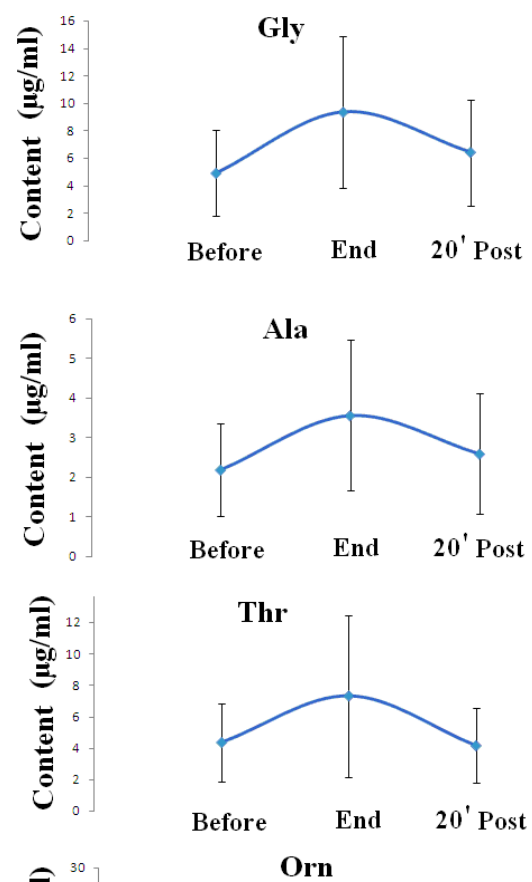

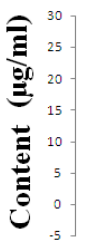

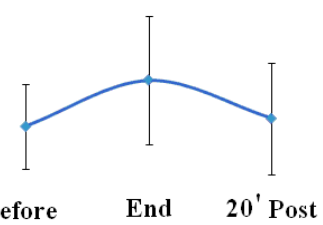

Figure 3.

The responsing curve of four kinds amino acids during stress experiment.

levels appear to increase during the vision stress experiment and to reduce after that. The results showed that salivary glycine, threonine, alanine, ornithine may potentially be another markers responding to psychological stress, though more comprehensive experiments are needed to support this conclusion.

\section{Acknowledgements}

This work was supported by the National Basic Research Program (No. 2012CB933302), the National Natural Science Foundation of China (No. 81172720, No. 21307086), and Colleges and universities in Jiangsu Province plans to graduate research and innovation projects (CXLX12_0121).

\section{REFERENCES}

Jones, D. P., Carlson, J. L., \& Samiec, P. S. (1998). Glutathione measurement in human plasma evaluation of sample collection, storage and derivatization conditions for analysis of dansyl derivatives by HPLC. Clinica Chimica Acta, 275, 175-184. http://dx.doi.org/10.1016/S0009-8981(98)00089-8

Kang, X. J., Xiao, J., Huang, X., \& Gu, Z.Z. (2006). Optimization of dansyl derivatization and chromatographic conditions in the determination of neuroactive amino acids of biological samples. Clinica Chimica Acta, 366, 352-356.

Nakamura, Y., Kodama, H., Satoh, T., Adachi, K., Watanabe, S., Yokote, Y. et al. (2010). Diurnal changes in salivary amino acid concentrations. Vivo, 24, 837-842.

Spencer, G.S. (1994). Hormone and metabolite changes with stress in stress susceptible pie train pigs. Endocrine Regulations, 28, 73-78.

Sun, J., Kang, X. J., Ma, Y. Q., Chen, L. Q., Qu, Z. J., Gu, Z. Z., \& Lu, Z. H. (2009). A potential quick determination used for monitor biological response of human under stress. 2009 the Regiomal Workshop of International Society for the Study of Behavioural Development (ISSBD), Nanjing, June 2009, 119-121.

Sun, J., Zheng, S. L., Wang, Y., Kang, X. J., Gu, Z. Z., \& Lu, Z. H. (2010). The preliminary investigation of salivary L-histidine under computer vision stress. The 7th International Conference on Cognitive Science (ICCS2010), Beijing, 17-20 August 2010, 394.

Yan, S. L., Zhao, G., \& Liu, Y. L. (2003). High-performance liquid chromatographic method for the determination of dansyl-amino acids. Journal of Capital Medical University, 24, 338-339. 